\title{
Artículos
}

\section{Tendencias que presentan las bibliotecas de ciencias de la salud}

\author{
Por Mercedes Rodríguez del Castillo Martín
}

\begin{abstract}
Resumen: En los últimos tiempos las bibliotecas en general, y las especializadas en ciencias de la salud en particular, están experimentando una evolución sin precedentes a lo largo de su historia. En el caso de estas últimas la proliferación de la información, los avances en biomedicina, el auge de las tecnologías y, muy especialmente, la expansión de internet han transformado los sistemas de acceso a la información provocando además una serie de conjeturas sobre su futuro. Los profesionales que las gestionan han visto la necesidad de replantearse la forma de organizar sus recursos para adaptarlos a la realidad actual. De este modo, aspectos como la forma de ampliar y mejorar los servicios que prestan a sus usuarios, la gestión de la información, la utilización de la Red como medio para acceder a la información y también intensificar la relación entre distintas bibliotecas con el fin de compartir recursos, el establecimiento de las intranets para la gestión de la información en las organizaciones, el uso de soportes digitales y la combinación de los sistemas tradicionales junto con los más recientes, y el papel del bibliotecario, cada vez más volcado en sus facetas como gestor de la información $y$ docente, constituyen elementos claves en las tendencias que presentan las bibliotecas de ciencias de la salud en la actualidad.
\end{abstract}

Palabras clave: Bibliotecas de ciencias de la salud, Tendencias, Servicios, Gestión del conocimiento, Bibliotecas digitales, Consorcios de bibliotecas.

\section{Title: Trends in health sciences libraries}

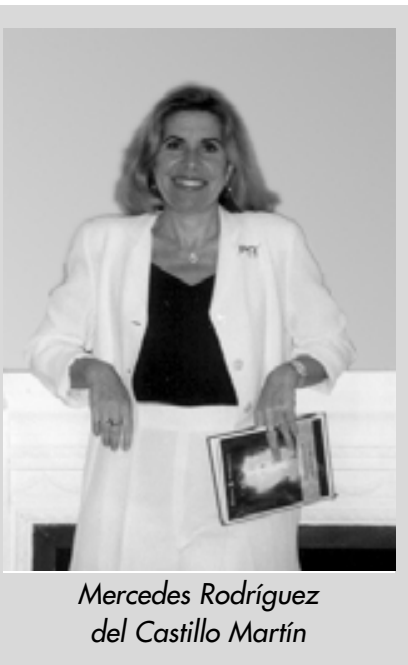

Abstract: In recent years libraries in general —and those specialized in health sciences in particular- are experiencing an unprecedented evolution In the case of the latter, developments in biomedical research and the ever-expanding body of biomedical knowledge, electronic resources and, most significantly, the expansion of the internet have transformed information access systems. Health sciences librarians have had to reconsider how to organize their resources in order to adapt them to the current situation. As a result, aspects such as the enlargement and improvement of user services, knowledge management and efficient literature searching, the use of internet for improving information access and interlibrary cooperation, the mix of traditional with new systems, and the librarian's role as both information agent and trainer, constitute key elements in the trends of health sciences libraries today.

Keywords: Health sciences libraries, Trends, Services, Knowledge management, Digital libraries, Libraries consortia.

Mercedes Rodríguez del Castillo Martín. «Tendencias que presentan las bibliotecas de ciencias de la salud». En: El profesional de la información, 2000, diciembre, v. 9, n. 12, pp 4-12.

\section{Las bibliotecas de ciencias de la salud}

Están integradas por las de hospitales, facultades de medicina, escuelas de enfermería, institutos y centros de investigación en biomedicina, las nacionales de medicina, escuelas de salud pública, facultades o instituciones farmacéuticas, veterinarias y en general todas aquellas cuyo fin es suministrar información relativa a las ciencias de la salud y a los profesionales de estas disciplinas para el desarrollo de su trabajo. Aparte de las peculiaridades propias relacionadas con el tipo de institución a la que pertenezcan, son muchas las características generales comunes a todas ellas: el tipo de fondos, sistemas de acceso a la información, servicios que prestan, etc.

El presente trabajo podría referirse a las bibliotecas de ciencias de la salud en general. No obstante, en algunos aspectos, el énfasis está dirigido a las de hospital que poseen una particularidad añadida: además de apoyar con la información necesaria a la investigación y docencia que se realiza en su institución tienen como misión principal ofrecer los datos necesarios para ayudar a mejorar la calidad del cuidado del paciente. A este respecto se perseguirían dos objetivos: el primero, de forma cotidiana, actualizando continuamente los conocimientos de los profesionales y el segundo aportando la información necesaria en casos clínicos concretos para ayudar a establecer un diagnóstico, un tratamiento, etc. Esto hace que las bibliotecas de hospital deban funcionar con criterios de exhaustividad, eficiencia, rapidez y, sobre todo, con un control riguroso en la calidad del material que suministran.

En cualquier caso cabría apuntar el hecho de que la denominación bibliotecas de ciencias de la salud va desplazando en muchas ocasiones al de bibliotecas médicas que podría implicar exclusividad o predominio de información dirigida a médicos o estudiantes de 
medicina. También hay que tener en cuenta que en la actualidad la propia escena de la medicina está cambiando; los límites entre ésta y otras disciplinas afines no son susceptibles de ser definidas tajantemente y las ciencias de la salud agrupan un amplio número de profesionales.

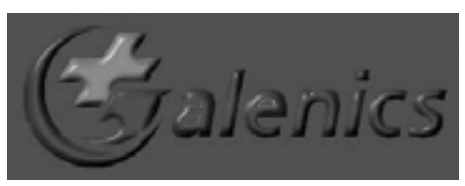

\section{Situación actual}

Durante los últimos veinte años estos centros han experimentado muchos cambios. Algunas de las circunstancias sociales que han contribuido a configurar las bibliotecas en las dos últimas décadas son: la proliferación de la información, la tecnología informática y las presiones económicas; incluyendo en estas últimas el aumento del precio de las publicaciones y la implantación y mantenimiento de las infraestructuras tecnológicas ${ }^{1}$. A partir de los años noventa con la aparición de internet se ha acelerado el proceso de transformación: nuevos mecanismos de acceder y recuperar la información están modificando la forma en que los bibliotecarios realizan sus tareas y organizan sus servicios.

La tecnología ha irrumpido de forma avasalladora en el mundo de la información y, a menudo, ha provocado cierta confusión en los conceptos llegando a extremos, para los no expertos en la materia, de confundir información con conocimiento y tecnologías con información; e incluso a hacerse la pregunta: ¿para qué se necesita una biblioteca hoy si todo es virtual?2.

Sin embargo la reacción en contra de las predicciones más agoreras ha ido dirigida no hacia la postración y el decaimiento de las bibliotecas, sino hacia una necesidad de adaptación, consecuencia de la actual demanda por parte de sus usuarios que cada vez les solicitan más información y servicios de mayor calidad ${ }^{3}$. Por otra parte, es obvio que la misión de estas bibliotecas no ha variado: continúa siendo facilitar la información necesaria a los profesionales sanitarios para el desarrollo de la práctica asistencial, la docencia y la investigación.

Al contemplar el panorama actual puede deducirse que, al menos a corto y medio plazo, van a continuar con la trayectoria de los años anteriores aunque, por supuesto, modificadas por las nuevas herramientas que facilitarán el acceso a la información en todos los aspectos añadiendo exhaustividad, calidad y rapidez. Si en el pasado fueron consideradas como una entidad física donde se concentraba la información ahora han pa- sado a ser un agente activo de la misma. Han eliminado sus barreras físicas y forman un entramado de servicios en el que los bibliotecarios cooperan entre ellos con más medios que antaño para acceder a esta información y seleccionarla. La idea de la biblioteca sin muros comienza a extenderse durante los años ochenta como resultado de las nuevas redes y sus tecnologías.

De otro lado, gracias a la lenta pero progresiva implantación del modelo de gestión del conocimiento, la biblioteca adquiere un papel integrador, otra tendencia que se evidencia claramente dentro de las organizaciones en donde los diferentes departamentos, instituciones relacionadas, etc., trabajan unidos creando un entorno conjunto de información. En el último Congreso internacional de bibliotecas médicas ${ }^{4}$ celebrado el pasado mes de julio en Londres, participaron representantes de todo el mundo compartiendo los mismos puntos de vista desde una perspectiva global aunque, por supuesto, con realidades materiales diferentes. Es cierto que salvo casos puntuales ningún país presenta la situación privilegiada de sus bibliotecas médicas como Estados Unidos y nadie puede negar la repercusión extraordinaria de su Biblioteca Nacional de Medicina en el resto del mundo, cuyo Index medicus, más tarde Medline, ha permitido el acceso a la información científica a todos los profesionales sanitarios de la comunidad internacional ${ }^{5}$.

\section{«Es de destacar la situación en el Reino Unido, que ha aposta- do fuertemente por sus bibliote- cas y bibliotecarios médicos co- mo parte activa en la práctica y difusión de la medicina basada en la evidencia»}

También es de destacar la situación en el Reino Unido que, a través de instituciones como el $U K \mathrm{He}$ alth Centre, ha apostado fuertemente por sus bibliotecas y bibliotecarios médicos como parte activa en la práctica y difusión de la medicina basada en la evidencia $(\mathrm{MBE})^{6}$. Pero en general, las técnicas y sistemas actuales necesarios para ofrecer servicios bibliotecarios especializados en ciencias de la salud se han difundido por todo el ámbito internacional. Incluso los bibliotecarios especializados de las naciones en vías de desarrollo participan de las mismas inquietudes que sus compañeros de países avanzados, contando en ocasiones con la ayuda de organismos como la Organización Mundial de la Salud'. Otra cosa sería su disponibilidad de recursos.

http://www.ihs.ox.ac.uk/library/homepage.htm 


\section{Tendencias. Líneas generales}

Hasta aquí es fácil dejarse seducir por todas las innovaciones que han invadido el mundo de la información en los últimos tiempos. Pero no hay que olvidar que todo proviene de los sistemas tradicionales y todos los recursos (los actuales y aquellos que desde siempre han servido para sustentar el conocimiento) serán, siempre en su totalidad, los que ofrezcan un eficaz servicio a la comunidad. Lo que hará valiosas las bibliotecas será su capacidad de suministrar conocimiento, es decir: información seleccionada, organizada, estructurada y rigurosa, no simples datos. Según el panorama que se ha intentado esbozar, todo hace pensar que las bibliotecas de ciencias de la salud se inclinarán hacia el desarrollo de los siguientes aspectos:

- Habrán de intensificar su misión como proveedores de servicios.

- La figura del bibliotecario se potenciará. Como experto en la gestión de la información pondrá en práctica sus conocimientos no sólo en las tareas de búsqueda y recuperación de información sino que elaborará y utilizará sistemas para su filtrado y selección e instruirá a otros profesionales en estas técnicas.

- Las bibliotecas se potenciarán gracias a poder enlazarse unas con otras aprovechando los sistemas de telecomunicaciones.

- Se ampliará el número de sus usuarios a través del acceso electrónico.

—No se deberán olvidar los sistemas y soportes tradicionales.

1. Las bibliotecas como proveedores de servicios. El médico en particular, y el resto de profesionales sanitarios en general, utilizan junto con otros medios las revistas científicas para su puesta al día. Éstas constituyen el soporte óptimo de información científica contrastada y fiable, aquella procedente de la investigación ${ }^{8}$. En la actualidad se publican anualmente alrededor de 25.000 títulos dedicados a las ciencias de la salud $^{9}$. Tansey calcula que anualmente aparecen 2.000.000 artículos sobre biomedicina. Es evidente que ningún profesional sanitario puede acceder físicamente a todas estas publicaciones, leerlas todas ni, por supuesto, evaluarlas ${ }^{10}$.

Por otra parte, la tecnología ha conseguido que el usuario final acceda individualmente a un amplio volumen de información desde cualquier punto. La Red ha derrumbado los muros de las bibliotecas y potencialmente suministra las herramientas necesarias para acceder a la información. Sin embargo la realidad es mucho más compleja. En un artículo aparecido en el British Medical Journal, Gray y Luisignan afirman que los profesionales sanitarios se enfrentan en mu- chas ocasiones al problema de sentirse abrumados por el actual volumen de información y no poder encontrar la apropiada en el momento y en el lugar que la nece$\operatorname{sitan}^{11}$.

Básicamente los buscadores y enlaces de hipertexto suministran herramientas de fácil utilización para recuperar documentos y el lector puede navegar de uno a otro. Sin embargo, es al final de su sesión cuando se puede evaluar el trabajo realizado teniendo en cuenta el tiempo que ha consumido, así como la calidad y pertinencia de los resultados de su búsqueda. Por esto, cada vez se hace más evidente la necesidad de que el el acceso no sea simplemente electrónico, sino intelectual, es decir, no va a ser suficiente suministrar datos, sino respuestas.

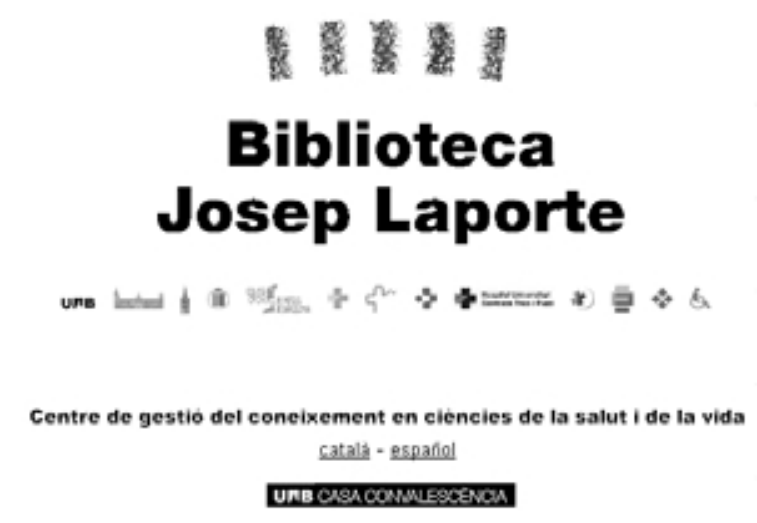

Cada día es más imprescindible observar conceptos como calidad de la información o gestión del conocimiento. La biblioteca deberá estar capacitada para facilitar información depurada y dirigida a necesidades concretas utilizando tecnologías (correo electrónico, etc.) que amplíen el radio de acción de sus servicios y al mismo tiempo permitan personalizarlos. Las bibliotecas usarán información proveniente de sus propios archivos, de su institución, y la descargarán desde recursos remotos, elaborarán datos y generarán conocimiento con el que ayudarán a atender a las necesidades específicas de sus usuarios. También ofrecerán la posibilidad de acceso a otras bibliotecas ${ }^{12}$.

Va dejando de ser un lugar donde yace la información. Sus fondos y recursos en general estarán cada vez más accesibles a todo el que pueda necesitarlos a través de sus páginas web, catálogos online, enlaces, etc., así como los catálogos colectivos situados en la web. Además la biblioteca deberá estar conectada por medio de una red local o intranet a los distintos departamentos que componen la institución a la que pertenece.

Igualmente, entre sus servicios deberá contar con equipos para la visualización de revistas en formato electrónico sin olvidar, por supuesto, las colecciones en soportes tradicionales (publicaciones periódicas, li- 
bros y literatura gris). A los habituales servicios de suministro del documento (reprografía tradicional o fotocopia y fax) se les añade sistemas telemáticos para la transferencia del documento o la utilización de una cuenta de la biblioteca para entregar los resultados de las búsquedas realizadas en las bases de datos. Otro recurso muy útil lo constituye la conexión de los catálogos a las revistas electrónicas.

\section{«Los profesionales sanitarios se enfrentan en muchas ocasio- nes al problema de sentirse abrumados por el actual volu- men de información y de no po- der encontrar la apropiada en el momento y en el lugar que la necesitan»}

También habrá que tener en cuenta que el horario de apertura de la biblioteca a sus usuarios debe ser tan amplio como sea posible y que es preciso continuar con otros procedimientos tradicionales como préstamo de libros, consulta en sala, etc. En el caso de los profesionales en medicina la biblioteca de su centro es el lugar idóneo para adiestrase en la utilización de internet conociendo, con el asesoramiento del bibliotecario, los mejores recursos de la web y dónde puede localizar la información que dé respuesta a sus necesidades ${ }^{13}$.

2. El bibliotecario como gestor de la información. En las normas de la Joint Commission on Accreditation of Health Care Organizations se hace la siguiente definición: la información basada en el conocimiento incluye documentación de revistas, información de referencia y datos de investigación y puede encontrarse en formas diversas, artículos, extractos o índices en formato impreso o electrónico, otras fuentes profesionales, etc. ${ }^{14}$.

En los últimos años, con la aparición de la MBE, se ha abundado en las tareas de seleccionar información para ser utilizada en la toma de decisiones clínicas. En realidad esta práctica, que existe desde hace años en la figura del bibliotecario clínico, ahora se expande y perfecciona con la elaboración de técnicas de criba y selección de información así como con criterios metodológicos de investigación clínica que permiten al médico recuperar selectivamente la apropiada para aplicarla al cuidado de sus pacientes ${ }^{15}$.

En este sentido, la misión del bibliotecario sería suministrar la información filtrada con arreglo a unos parámetros de calidad y enfocada a casos específicos para ayudar al médico en sus tomas de decisión, así como ofrecer información de calidad para redactar guías de actuación, protocolos y cualquier actividad dentro de la práctica sanitaria. El bibliotecario debe poner énfasis especial en su tarea como proveedor de información depurada, rigurosa y precisa.

En los últimos tiempos, y de forma generalizada, los bibliotecarios de hospital se han interesado en familiarizarse y dominar conceptos referidos a la investigación médica. El bibliotecario-documentalista médico no es sólo un organizador y difusor de la información, sino que se ha esforzado en entender la literatura que gestiona. Ha comprendido la necesidad de aprender sobre las distintas clases de estudios y ensayos concernientes a la investigación clínica y de este modo está capacitado para ayudar a los médicos a identificar y recuperar información con destino a los cuidados de pacientes ${ }^{16}$.

Hoy existe gran cantidad de recursos de información para la MBE disponibles a través de los soportes tradicionales o de internet. Por señalar algún ejemplo significativo: la biblioteca digital del UK Health Centre, la sección Clinical Queries de Pubmed, la Cochrane Library y otros muchos; véase al respecto el trabajo de Bravo y Campos "Cómo hacer una búsqueda bibliográfica en internet" ${ }^{\prime \prime}$. Sin embargo, el facultativo puede encontrarse con dificultades para acceder a la información rigurosa a la que se alude. Algunos de estos problemas podrían ser: falta de tiempo para hacer una revisión completa, el desconocimiento de todas las fuentes disponibles, la cuestión de los lenguajes documentales, las complejas estrategias de búsqueda, etc. El bibliotecario domina todos estos conocimientos y destrezas y con ellas ayudará al profesional sanitario para encontrar la información.

http://www.ncbi.nlm.nih.gov/Pubmed/

http://www.update.software.com/ccwe/cochrane/cdsr. html

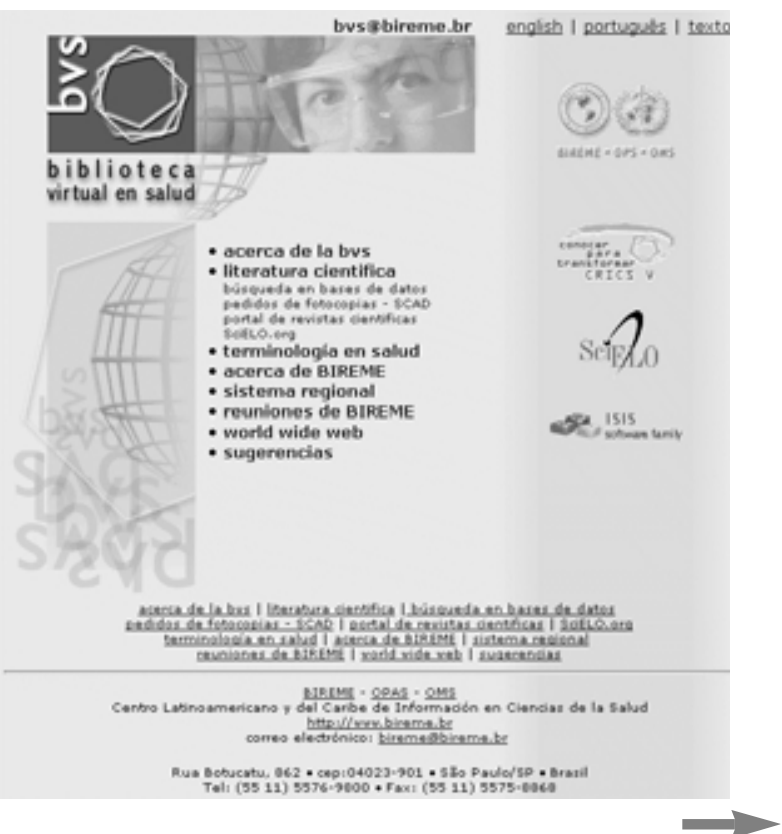

El profesional de la información, vol. 9, no 12, diciembre 2000 
La importancia y vigencia de este aspecto en la tarea actual del bibliotecario médico queda demostrada en el relieve que se ha dado al tema en el octavo y último Congreso internacional de bibliotecas médicas celebrado en Londres el pasado mes de julio referido anteriormente.

3. El bibliotecario como instructor de técnicas y sistemas de acceso al conocimiento. El aspecto docente de los bibliotecarios médicos se ha intensificado en los últimos años. En épocas ya lejanas enseñaron a sus usuarios a manejar Index medicus, Excerpta medi$c a$, etc.; más tarde los familiarizaron con las conexiones en línea para el acceso a las bases de datos y, con la aparición del cd-rom a finales de los años ochenta, se generalizó el hábito de instruirlos en la búsqueda y recuperación de información en este soporte. En la actualidad, como se ha dicho, la biblioteca es el lugar apropiado para conocer los recursos y sistemas para navegar por la Red con vistas a conseguir los mejores resultados. El bibliotecario ahora tiene como una de sus actividades más importantes la de enseñar al personal sanitario a localizar información de calidad. Así, podrá utilizar complejas estrategias de búsqueda para llevar a cabo revisiones sistemáticas, seleccionar ensayos clínicos o cómo se han de usar los lenguajes documentales para conseguir información rigurosa y con$\operatorname{creta}^{18}$.

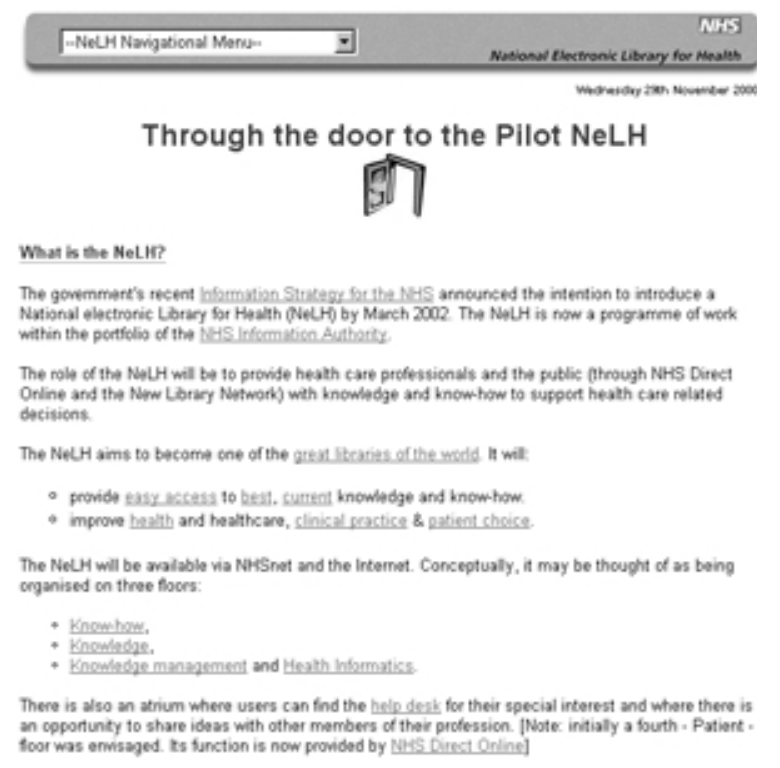

\section{La expansión de las bibliotecas: bibliotecas digitales. Consorcios}

1. Las bibliotecas digitales. En los últimos años el término biblioteca se ha visto modificado en múltiples ocasiones con el adjetivo de virtual o digital. Podría pensarse que, quizás debido al temor de presentar una imagen desfasada, muchos han creído oportuna la adopción de nuevos nombres o adjetivos que ofrezcan otro aspecto, más actualizado e innovador. En su artí- culo "The digital library: an oxymoron?"19 Guédon realiza un brillante análisis del desarrollo de la comunicación y de la distribución de la información. Considera que no es necesario hacer referencia al continente en donde la información se almacena si bien, afirma, esto no quiere decir que el contenedor no haya influido y lo continúe haciendo en la arquitectura de la biblioteca y en las tareas que deban desarrollar los profesionales que en ella trabajan. ¿Significa esto que se está prestando excesiva importancia a las nuevas herramientas y soportes que deben utilizarse y se pierde de vista la auténtica finalidad del trabajo informativo que se desarrolla en las bibliotecas?

\section{«Se hace más evidente que no sirve el simple acceso electróni- co si no va acompañado de co- nocimiento intelectual, es decir, no es suficiente suministrar da- tos sino respuestas»}

En realidad, y aunque no siempre las palabras resulten inocentes, para muchos el adjetivo digital es superfluo. Las bibliotecas han ido adaptando a lo largo de su historia los medios que iban apareciendo en su misión de conservar y transmitir el conocimiento y ésta es la perspectiva que no debe abandonarse. La biblioteca de ciencias de la salud actual debe ofrecer a sus usuarios un acceso fácil al conocimiento, ya estructurado y organizado por los expertos de manera que encuentren la información pertinente y de calidad en el modo y tiempo que necesitan. Así facilitarán a estos profesionales la información óptima para la práctica clínica.

Existe gran cantidad de bibliotecas electrónicas que ofrecen servicios muy útiles. De entre los múltiples ejemplos que cabría citar, mencionaremos la $\mathrm{Na}$ tional Electronic Library for Health, del Institute of Health Science de Oxford en el Reino Unido. Dispone de un buscador, enlaces y servicios de alerta para información individualizada. Contiene información para MBE, guías de práctica clínica e información para el paciente. Ha puesto un énfasis especial en el aspecto de la gestión del conocimiento desarrollando tareas y recursos necesarios para su producción, búsqueda y distribución. Otro ejemplo europeo es el hospital de Rouen en Francia.

http://www.nelh.nhs.uk

\section{http://www.chu-rouen.fr/dsii/html/interdef.html}

Las bibliotecas que no disponen de los medios suficientes para aportar de modo tan amplio sus servicios a la comunidad están realizando también un esfuerzo notable dentro de sus posibilidades incorporando los 
sistemas actuales que les permiten trascender sus barreras físicas. De esta manera muchos centros, cuyos recursos son mucho más limitados, utilizan los métodos electrónicos y se sirven de estas grandes bibliotecas, y de la información que ofrecen, actuando de enlaces para sus usuarios, elaborando guías, listas de direcciones de interés y buscando en definitiva, de entre los recursos que ofrece la Red, aquellos que pueden ser de mayor utilidad a sus usuarios; los organizan y los ponen a su disposición para que puedan atender a sus necesidades de información de la manera más eficaz posible.

2. Consorcios de bibliotecas. Han venido existiendo desde décadas atrás con el objetivo de compartir recursos, tanto humanos como financieros. Comenzaron en los EE.UU. a partir de los recortes presupuestarios de los años setenta, y más tarde esta práctica se extendió al resto del mundo. No existen dudas de que con el advenimiento de internet y la web se ha potenciado la innovación de modos y la proliferación de sistemas de información y bases de datos, así como la posibilidad de acceder a las colecciones de las diferentes bibliotecas que componen un consorcio.

El establecimiento de redes y las intranets presentan unas nuevas herramientas para las asociaciones y la colaboración entre bibliotecas. Tradicionalmente, las iniciativas de cooperación se centraban especialmente en el préstamo interbibliotecario. $\mathrm{El}$ ejemplo de EE.UU. es seguramente el más notable. Una vez más, es este país el que toma la iniciativa al desarrollar nuevos sistemas y mejores recursos en el ámbito de las bibliotecas de ciencias de la salud. Pueden citarse como ejemplos los siguientes: el Health Sciences Libraries Consortium ( $S L C)$, una organización sin ánimo de lucro fundada para pro-

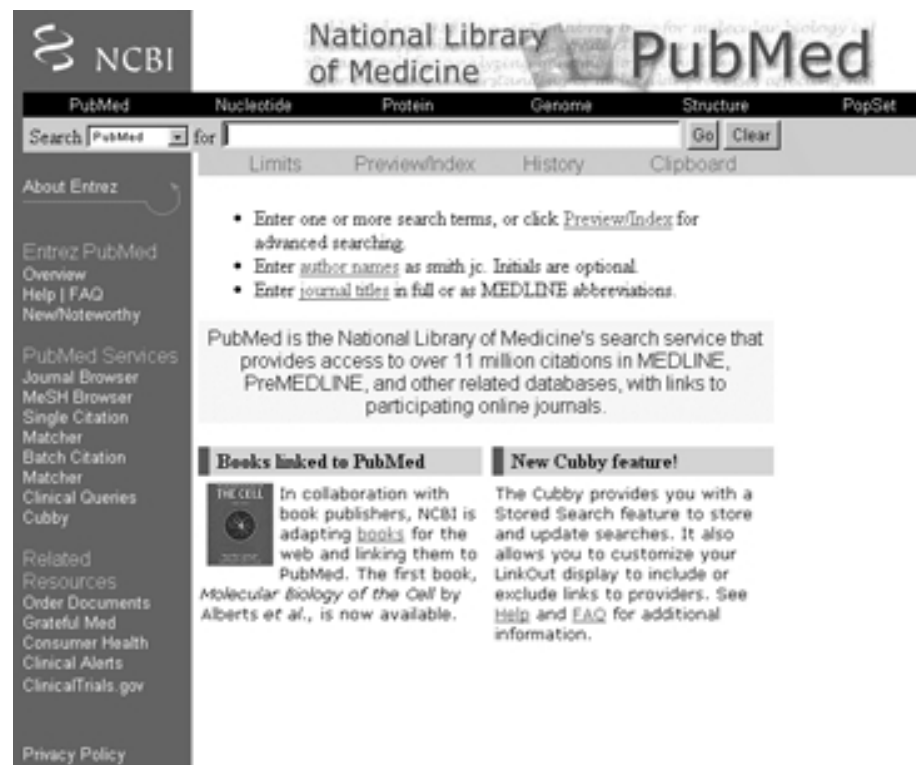
mover la información y que tiene entre sus miembros al Colegio Médico de Filadelfia, la Universidad de Ciencias de la Salud Alleghemy, la Universidad Penn State, la Universidad Thomas Jefferson, la de Pensilvania, así como alrededor de 500 miembros asociados de varios estados.

http://www.hscl.org

Otro ejemplo de cooperación interbibliotecaria es el convenio establecido en 1997 para la adquisición de servicios de suscripciones de revistas que incluía 17 hospitales en Missouri, Ocklahoma, Arkansas y Texas. También cabría citar el consorcio constituido por los hospitales de Nueva York y la Red de Bibliotecas de la Universidad de Búfalo (Hubnet), un sistema que suministra bases de datos, información médica a texto completo y servicios de internet con conexiones directas desde las bibliotecas, los distintos servicios del hospital y consultas médicas durante las 24 horas.

http://www.bgh.edu/library/hubmain.htm

Otros ejemplos dignos de mención son: la Red de Información Sanitaria del Estado de Arizona (Azhin), el área de Rochester en Nueva York (Miraclenet), o el Consorcio de Bibliotecas de Ciencias de la Salud de Pensilvania ${ }^{20}$.

http://www.azhin.org

http://www.urmc.rochester.edu/miraclenet/

http://www.hslc.org

Estas redes utilizan internet como un vehículo para enlazar a sus clientes colectivos con el conocimiento médico que tradicionalmente se hacía a través de las bases de datos pero que hoy se lleva a cabo utilizando el texto completo de las revistas seleccionadas y las plataformas interactivas. También son habituales las suscripciones colectivas de recursos online que reducen la dependencia del préstamo interbibliotecario a través de la adquisición de colecciones a texto completo. Como es lógico, no podrían enumerarse aquí los múltiples y relevantes ejemplos de redes y consorcios que funcionan en la actualidad dentro del área de ciencias de la salud. Pero no puede dejar de mencionarse Bireme, que continúa su trayectoria iniciada años atrás y que ha conseguido expandir y mejorar su actuación agrupando a los países del ámbito iberoamericano y del Caribe.

\section{http://www.bireme.br}

En España, desde hace algún tiempo se están desarrollando diferentes actividades de colaboración entre bibliotecas médicas en varias comunidades autónomas. El Servicio Gallego de Salud ha reunido 17 de sus más importantes hospitales con el objetivo de uni- 
ficar funciones como distribución de bases de datos, descripción bibliográfica, traducción de encabezamientos de materias médicas, elaboración de guías, etc. En Cataluña existe la Fundación Biblioteca Josep Laporte, un consorcio de bibliotecas médicas cuya misión es ofrecer a sus usuarios información especializada de calidad y que cuenta entre otros recursos con una biblioteca médica tradicional, un centro de teledocumentación, una biblioteca digital con bases de datos, revistas a texto completo y productos basados en la síntesis de la evidencia. También en Cataluña se encuentra Galenics, una fundación que funciona como consorcio que cuenta con un metaservidor, un nodo neutral e instrumentos para formación y, a través de acuerdos, cualquier institución del área sanitaria puede formar parte de la misma.

http://www.fbjoseplaporte.org

http://www.galenics.com

En Madrid se ha llevado a cabo un estudio de la situación dentro de un proyecto de colaboración de bibliotecas de ciencias de la salud en esta comunidad. En el País Vasco existe, auspiciado por el Servicio Vasco de Salud, un diseño de red cooperativa para el uso de publicaciones periódicas, bases de datos, acceso electrónico al catálogo unificado, etc. En Andalucía se está llevando a cabo un estudio con vistas a la implantación de una red para compartir recursos en el área de estas bibliotecas de la comunidad (para mayor información sobre el tema, véase la ponencia de Ribes, García y Rodríguez del Castillo: "Collaboration among Spanish medical libraries", presentada en el último Congreso internacional de bibliotecas médicas ${ }^{20}$.

\section{«La biblioteca de ciencias de la salud debe ofrecer a sus usua- rios un acceso fácil al conoci- miento, ya estructurado y orga- nizado por los expertos docu- mentalistas»}

Por otra parte, y aludiendo al aspecto integrador que se mencionaba anteriormente como otra de las tendencias que presentan estas bibliotecas, podría citarse otro modo de cooperación interbibliotecaria como el constituido por el Integrated Advanced Information Management Systems (Iaims). Este proyecto fue fundado en los ochenta por la Biblioteca Nacional de Medicina de los EE.UU. Años después de su creación, Klein lo describe como un esfuerzo para integrar la información de las historias clínicas de los pacientes en un programa de biblioteca clínica y otros servicios de referencia electrónica con acceso a la red regional de bibliotecas automatizadas ${ }^{22}$.

\section{Ampliación del número de usuarios}

Es clara la tendencia de que las bibliotecas aumentan el número de sus usuarios potenciales ya que, aparte de los que accedan a ella de forma tradicional, pueden serlo todos aquellos a los que pueda facilitar información a través de distintos sistemas digitales: catálogos online, páginas web, archivos propios y cualquier otra fuente de información que la biblioteca ofrezca a sus usuarios más remotos.

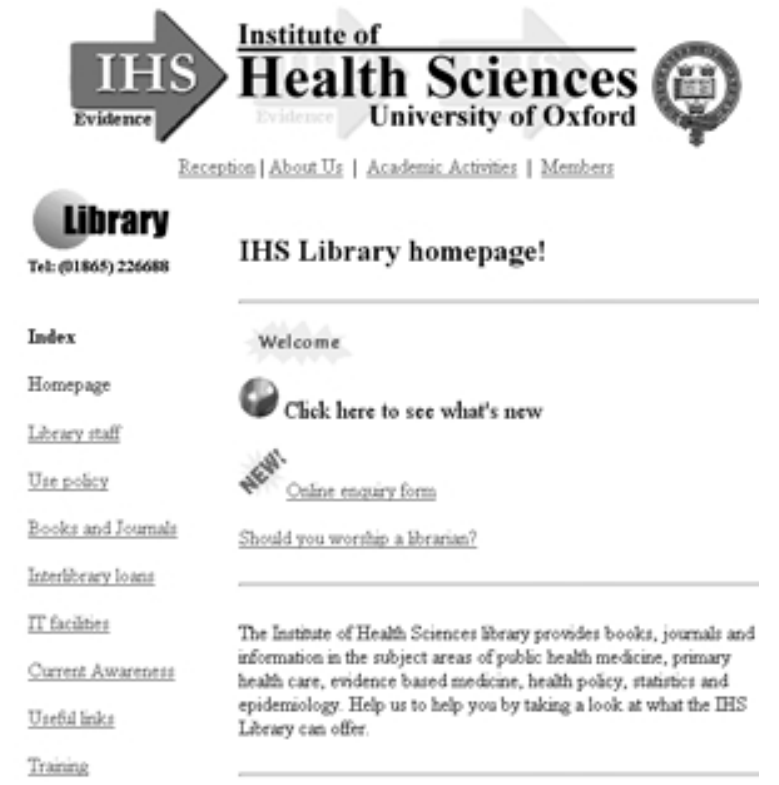

Además, si una de las mejores características de la Red es su capacidad de democratizar el conocimiento no hay duda que el mejor lugar para ofrecer este servicio a la comunidad es desde la biblioteca, accesible a cualquier persona, profesionales e incluso pacientes o familiares que precisen información. No todo el mundo tiene la posibilidad de disponer un acceso personal y los sistemas necesarios. La biblioteca cumple con su misión de siempre, de facilitar a todos los que lo puedan necesitar el acceso a la información y siempre que sea requerido, asistido e instruido por profesionales de la información ${ }^{23}$.

1. Los sistemas tradicionales. $\mathrm{Al}$ menos a medio plazo parece evidente que seguirán existiendo los recursos tradicionales: libros, revistas, bases de datos, etc., aparte de las publicaciones electrónicas, archivos de sonidos, imágenes y demás. En muchos casos el acceso electrónico a la información ha sido visto por parte de la administración como una solución que permitiría ahorrar costos, tanto en lo que se refiere a la adquisición de fondos, como un medio para paliar el eterno problema de falta de espacio. En realidad estas expectativas no parece que actualmente se vayan a cumplir.

En primer lugar habrá que detenerse a considerar si la documentación que necesitan los usuarios habituales está toda accesible en formato electrónico. Es 
evidente que no. El porcentaje de publicaciones médicas disponibles a través de internet es hoy por hoy bastante limitado a pesar del aumento que pueda producirse en un futuro próximo. Michele Klein realizó un ilustrativo trabajo al respecto donde describe el bajo porcentaje de revistas indexadas en Index medicus que están disponibles a texto completo en edición electrónica y que aun de esas, un gran número de editores requiere también suscripción en papel ${ }^{24}$. Al principio de la utilización de estos sistemas miembros de las instituciones no especializados en el tema pensaron que todo era gratis en la Red. Esto contribuyó a crear esas falsas expectativas para abordar la forma de organizar las bibliotecas en un futuro concibiendo esperanzas de que podría ahorrarse espacio y dinero.

En lo que se refiere a la cuestión del espacio de momento al menos, las bibliotecas no disminuyen su tamaño físico ${ }^{25}$. Ahora, lo que están experimentando es la necesidad de acondicionar lugares para instalar los equipos para el acceso electrónico y también donde los bibliotecarios puedan instruir a sus usuarios en las técnicas de acceso y recuperación de la información. No olvidemos además la misión que deben cumplir como conservadores del conocimiento. Obsesionados por el valor de la actualidad puede incurrirse en el descuido de olvidar la tarea de preservar información valiosa que puede ser recabada en un futuro más o menos lejano ${ }^{26}$.

\section{«La denominación bibliotecas de ciencias de la salud va des- plazando en muchas ocasiones al de bibliotecas médicas que podría implicar exclusividad o predominio de información diri- gida a médicos o estudiantes de medicina»}

Para muchos, la tecnología no debe entenderse como la solución para el ahorro de espacio sino como un recurso aparte. Así lo documentan Sheldlock y Ros en su estudio aludido anteriormente donde llegan a la conclusión de que, en lugar de solucionar la necesidad de espacio la tecnología necesita su propio espacio. La biblioteca actual deberá ser una mezcla de sistemas nuevos y tradicionales para adaptarse a las diferentes

\section{Próximos números monográficos}

Marzo 200I Hosts y productores de bases de datos Mayo 200I Información para la inteligencia competitiva

Septiembre 200I Archivos

Los interesados pueden remitir notas, artículos, propuestas, publicidad, comentarios, etc., sobre estos temas a:

$$
\text { epi@sarenet.es }
$$

necesidades de sus usuarios. Junto con los métodos de acceso electrónico se necesitarán fondos impresos y lugares adecuados para leer ${ }^{27}$. Es por todo esto que, sin duda, la realidad se presenta más compleja y todo hace pensar que habrá que jugar tanto con los modelos tradicionales como con los actuales, combinándolos en la mejor manera posible para cumplir con eficiencia su misión.

\section{Conclusiones}

Las consideraciones expuestas hacen pensar que, al menos en un futuro próximo, las bibliotecas de ciencias de la salud presentan las siguientes tendencias:

Están potenciando su imagen como proveedores de servicios. Descargarán información y realizarán sus propios archivos y harán revisiones sistemáticas de la literatura con destino al cuidado del paciente. Posiblemente en un futuro cada vez más próximo, y de forma más generalizada, el acceso no será simplemente para buscar datos sino respuestas. La gestión de la información y el concepto de su calidad deberán ser observados cuidadosamente. Cabe entender por tanto que los servicios no sólo facilitarán el acceso a los recursos de información, sino que proporcionarán evaluaciones de calidad y filtro, e información personalizada dirigida a gestores, directivos y especialistas.

Los servicios comprenderán todo aquello que cubra las necesidades de información, añadiendo algunos como el envío de la información (en papel, disquete o mediante correo electrónico), siendo necesaria la potenciación de sistemas de suministro electrónico del documento. Igualmente, en la actualidad se está incrementando la accesibilidad a la información por medio de los catálogos de los fondos a través de las intranets de las instituciones y los catálogos colectivos en la web. La red local en las organizaciones es un recurso cada vez más utilizado con la conexión de la biblioteca a las distintas secciones que componen el centro. Los distintos departamentos, instituciones relacionadas, etc., trabajando unidos crearán un entorno conjunto de información dentro de las organizaciones.

Cada vez es más evidente la necesidad de abundar en tareas de gestión y organización del conocimiento. La utilización de sistemas de selección y filtros de calidad de la información, la realización de revisiones sistemáticas de la literatura con destino al cuidado del paciente, etc., son funciones cotidianas para el bibliotecario médico. Es por esta razón que los bibliotecarios científicos están adquiriendo un notable 
auge en el ejercicio de sus tareas. Es necesario por tanto un personal cualificado capaz de recuperar, evaluar y sintetizar información basada en el conocimiento y personal de apoyo y auxiliar.

Es también clara la tendencia de que las bibliotecas crecerán al poderse enlazar cada vez en mejores condiciones a través de las conexiones digitales. De este modo ampliarán sus áreas de acceso (a mayor acceso mayor eficacia) y se elaborarán convenios de cooperación. Al igual que en los años ochenta se crearon redes de cooperación interbibliotecaria, se formarán consorcios de servicios bibliotecarios con lo que expandirán sus servicios y el número de sus usuarios.

No podrán desecharse, al menos en un futuro próximo, los recursos tradicionales: libros, revistas, bases de datos, etc. Deberá haber un espacio físico apropiado para la lectura y el estudio aparte de otro para las revistas electrónicas, archivos de sonidos, imágenes, etc. Con todo esto, las bibliotecas de ciencias de la salud seguirán cumpliendo con la misión que han tenido siempre. Los cambios que experimentan son simples modificaciones al adoptar los mejores recursos que el progreso ofrece, como hace el resto de la sociedad a la que sirven.

\section{Notas:}

1. Rodríguez del Castillo Martín, M. Las bibliotecas de ciencias de la salud. Estudio de la situación en los hospitales de España. Tesis doctoral. Universidad de Granada, 1996.

2. Weise, F. O. "The health sciences and human services library: this is one sweet library". En: Bulletin of the Medical Library Association, 1999, v. 87, n. 2, pp. 170-177.

3. Matheson N. W. "The idea of the library in the twenty-first century". En: Bulletin of the Medical Library Association, 1995, v. 65, n. 1, pp. 1-7.

4. Proceedings of the 8th international congress on medical librarianship, 2000. Consultado en agosto de 2000.

http://www.icml.org/confprocl

5. Smith, E. V.; Scott, F. J. "Exporting the American (information) revolution: the international impact of the National Library of Medicine". En: Bulletin of the Medical Library Association, 1986, v. 74, n. 4, pp. 339-343.

6. Murphy, J. "The role of health science librarians in preparing tomorrow`s doctors to manage information knowledge management in the NHS: positioning the healthcare librarian at the knowledge intersection". En: Health libraries review, 2000, v. 17, n. 1, pp. 7-13.

7. Pakenham-Walsh, N. "Access to reliable information for healthcare workers in developing countries: an international perspective". En: Health libraries review, 2000, v. 17, n. 1, pp. 37-40.

8. Gómez de la Cámara, A. Manual de medicina basada en la evidencia. Elementos para su desarrollo y aplicación en atención primaria. Madrid: Jarpyo Editores, 1998.

9. Cook, D.; Ellrodt, G. "The potential role of clinical guidelines in the ICI”. En: Current opinion in critical care, 1996, v. 2, pp. 326-330.
10. Tansey, E. M. "The dustbin of history, and why so much of modern medicine should up there". En: The lancet, 1999, 20 de noviembre, v. 354, n. 9.192, pp. 1811-1812.

11. Gray, J. M.; Lusignan L. de. "National Electronic Library for Health (Nelh)". En: British Medical Journal, 1999, v. 319, n. 7.223, pp. 14761479 .

12. Behm, L. M.; Combs, A.; Rattenborg, K. N.; Schell, E.; Vishwanatham, R. "Librarian's use of health web for reference and instruction". En: Medical reference service quarterly, 1999, v. 18, n. 1, pp. 1-10.

13. Means, M. L. "The research funding service. A model for expanded library services". En: Bulletin of the Medical Library Association, 2000, v. 88, n. 2, pp. 178-186.

14. The Joint Commission on Accreditation of Healthcare Organizations. Manual de acreditación para hospitales. Barcelona: SG Editores, 1996. Isbn 0-86688-444-0.

15. Rodríguez del Castillo Martín, M. "La información bibliográfica aplicada a la práctica médica”. En: El profesional de la información, 1998 , mayo, v. 7, n. 5, pp. 10-13.

16. Scherrer, C. S.; Dorsch, J. L. "The envolving role of the librarian in evidence based medicine". En: Bulletin of the Medical Library Association, 1999, v. 87, n. 3, pp. 322-3286.

17. Bravo Toledo, R.; Campos Asensio, C. "Cómo hacer una búsqueda bibliográfica en internet”. En: FMC Formación médica continuada en atención primaria, 2000, v. 7, n. 5, pp. 307-309.

18. McKibbon, A.; Eady, A.; Marks, S. PDQ evidence-based principles and practice. Hamilton: Decker Inc., 1999.

19. Guedon, J. L. "The digital library: an oxymoron?". En: Bulletin of the Medical Library Association, 1999, v. 87, n. 1, pp. 9-19.

20. Riordan, M. L.; Perry, G. J. "Interlibrary cooperation: from ILL to Iamims and beyond". En: Bulletin of the Medical Library Association, 1999, v. 87, n. 3 , pp. $251-255$.

21. Ribes Cot, F.; García Martín, M. A.; Rodríguez del Castillo Martín, M. "Collaboration among Spanish medical libraries". VIII International congress of medical librarianship, 2000. Consultado en agosto de 2000.

http://www.icml.org/confproc/

22. West, R. T. "Iaims: an interview with Dick West. Integrated advanced information management systems". En: Journal of American Medical Informatic Association, 1999, v. 6, n. 6, pp. 447-456.

23. Stephenson, J. "National Library of Medicine to help consumers use online health data". En: Jama, Journal of the American Medical Association, 2000, 5 de abril, n. 13, pp. 1675-1676.

24. Klein, M. S. "Balancing divergent expectations". En: Bulletin of the Medical Library Association, 1999, v. 87, n. 1, pp. 88-91.

25. Sheldlock, J.; Ros, F. "A library for the twenty first century: the Galter Health Sciences Library's renovation and expansion project”. Bulletin of the Medical Library Association, 1997, v. 85, n. 2, p. 176-186.

26. Fortney, L. "Thinking about replacing print biomedical journals with electronic? Think again". En: National network, 1997, v. 22, n. 1, pp. 6.

27. Peay, W. J. "Strategies and measures for our next century". En: Bulletin of the Medical Library Association, 1999, v. 87, n. 1, pp. 1-8.

Mercedes Rodríguez del Castillo Martín. Biblioteca general del Hospital Universitario Virgen de las Nieves, Granada. mercedes@hvn.sas.cica.es 